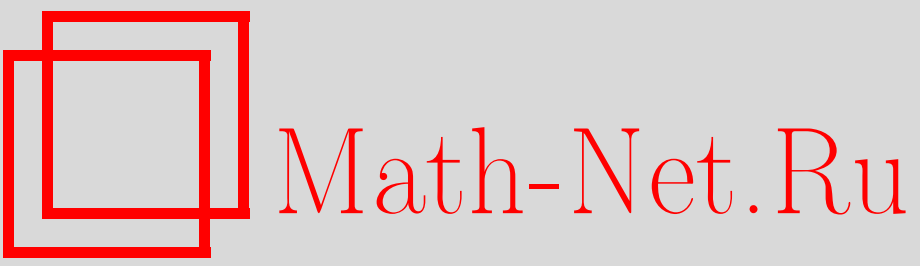

В. В. Соколов, А. В. Цыганов, Коммутативные пуассоновы подалгебры для скобок Склянина и деформации известных интегрируемых моделей, ТМ $\Phi, 2002$, том 133, номер 3, 485-500

DOI: https://doi.org/10.4213/tmf413

Использование Общероссийского математического портала Math-Net.Ru подразумевает, что вы прочитали и согласны с пользовательским соглашением

http://www.mathnet.ru/rus/agreement

Параметры загрузки :

IP: 107.22 .136 .117

26 апреля 2023 г., 16:24:04 


\section{КОММУТАТИВНЫЕ ПУАССОНОВЫ ПОДАЛГЕБРЫ ДЛЯ СКОБОК СКЛЯНИНА И ДЕФОРМАЦИИ ИЗВЕСТНЫХ ИНТЕГРИРУЕМЫХ МОДЕЛЕЙ}

Построены иерархии коммутативных пуассоновых подалгебр для скобок Склянина. Каждая из подалгеб порождается полным набором интегралов в инволюции. С использованием различных известных представлений скобок найдены новые интегрируемые системы и разделение переменных для них. Среди построенных моделей содержатся деформации волчка Горячева-Чаплыгина, цепочки Тоды и модели Гейзенберга.

Ключевые слова: конечномерные интегрируемые системы, представление Лакса, $r$-матричные алгебры, разделение переменных.

\section{1. ВВЕДЕНИЕ}

В работе рассматриваются известные скобки Склянина [1], [2] на множестве матриц вида

$$
T(\lambda)=\left(\begin{array}{ll}
A & B \\
C & D
\end{array}\right)
$$

с коэффициентами, полиномиально зависяшими от параметра $\lambda$. Определение скобок содержится в разделе 2 .

Для первых скобок Склянина коэффициенты матрицы $T(\lambda)$ имеют вид

$$
\begin{array}{ll}
A(\lambda)=\lambda^{N}+A_{N-1} \lambda^{N-1}+\cdots+A_{0}, & B(\lambda)=B_{N-1} \lambda^{N-1}+\cdots+B_{0} \\
C(\lambda)=C_{N-1} \lambda^{N-1}+\cdots+C_{0}, & D(\lambda)=D_{N-2} \lambda^{N-2}+\cdots+D_{0}
\end{array}
$$

Скобка Склянина задает структуру алгебры Пуассона на множестве $\mathfrak{A}_{N}$ всех функций, зависяших от коэффициентов

$$
A_{0}, \ldots, A_{N-1}, B_{0}, \ldots, B_{N-1}, C_{0}, \ldots, C_{N-1}, D_{0}, \ldots, D_{N-2}
$$

\footnotetext{
* Институт теоретической физики им. Л. Д. Ландау, Москва, Россия. E-mail: sokolov@landau.ac.ru

${ }^{\dagger}$ Санкт-Петербургский государственный университет, Санкт-Петербург, Россия. E-mail: tsiganov@mph.phys.spbu.ru
} 
матрицы $T(\lambda)$.

Для второй скобки Склянина коэффишиенты $T(\lambda)$ имеют следуюшую структуру:

$$
\begin{aligned}
& A(\lambda)=\bar{A}_{2 N} \lambda^{2 N}+\bar{A}_{2 N-1} \lambda^{2 N-1}+\cdots+\bar{A}_{0}, \\
& B(\lambda)=\bar{B}_{N-1} \lambda^{2 N-1}+\cdots+\bar{B}_{1} \lambda^{3}+\bar{B}_{0} \lambda, \\
& C(\lambda)=\lambda^{2 N+1}+\bar{C}_{N-1} \lambda^{2 N-1}+\bar{C}_{N-2} \lambda^{2 N-3}+\cdots+\bar{C}_{0} \lambda, \\
& D(\lambda)=A(-\lambda) .
\end{aligned}
$$

В этом случае образующими пуассоновой алгебры $\mathfrak{A}_{N}$ являются переменные

$$
\bar{A}_{0}, \ldots, \bar{A}_{2 N}, \bar{B}_{0}, \ldots, \bar{B}_{N-1}, \bar{C}_{0}, \ldots, \bar{C}_{N-1} .
$$

Для обеих скобок размерность симплектического листа обшего положения равна $2 N$.

Основным свойством скобок Склянина является то, что для некоторых специальных постоянных матриц $K(\lambda)$ коэффициенты следа матрицы $K(\lambda) T(\lambda)$ образуют $N$-мерную коммутативную подалгебру $\mathfrak{A}_{N}^{0}$ в $\mathfrak{A}_{N}$. Эта подалгебра порождена линейными многочленами переменных (1.3) или (1.5).

В этой статье мы строим другие $N$-мерные коммутативные подалгебры $\mathfrak{A}_{N}^{M}$ в $\mathfrak{A}_{N}$, $M \in \mathbb{N}$, порожденные многочленами $I_{1}^{M}, \ldots, I_{N}^{M}$ более высоких степеней, задающими набор $N$ интегралов в инволюции. Переменные разделения для всех этих наборов одни и те же, в то время как кривые разделения зависят от $M$.

Используя известные представления скобок Склянина и наши семейства интегралов в инволюции, мы строим новые интегрируемые модели и разделения переменных для них. Эти модели являются деформациями базисных моделей, соответствуюших подалгебре $\mathfrak{A}_{N}^{0}$, порожденной линейными интегралами. Наиболее интересные деформации связаны с коммутативной подалгеброй $\mathfrak{A}_{N}^{1}$, содержашей многочлен второй степени от переменных (1.3) или (1.5).

Один из примеров связан с представлением первых скобок Склянина на алгебре $e(3)$ [1], [3] со скобками Пуассона

$$
\left\{J_{i}, J_{j}\right\}=\varepsilon_{i j k} J_{k}, \quad\left\{J_{i}, x_{j}\right\}=\varepsilon_{i j k} x_{k}, \quad\left\{x_{i}, x_{j}\right\}=0, \quad i, j, k=1,2,3,
$$

где $\varepsilon_{i j k}$ - полностью кососимметрический тензор.

Оказывается, что построенная нами подалгебра $\mathfrak{A}_{2}^{1}$ соответствует квадратичной деформации [4] волчка Горячева-Чаплыгина с гамильтонианом

$$
\begin{aligned}
H= & J_{1}^{2}+J_{2}^{2}+4 J_{3}^{2}+2 \alpha_{1} x_{1}+2 \alpha_{2} x_{2}+\alpha_{3} J_{3}+ \\
& +4\left(\alpha_{4} x_{1}+\alpha_{5} x_{2}\right) J_{3}-\left(\alpha_{4}^{2}+\alpha_{5}^{2}\right) x_{3}^{2}+\frac{\alpha_{6}}{x_{3}^{2}} .
\end{aligned}
$$

На нулевом уровне интеграла площадей динамическая система с таким гамильтонианом является интегрируемой по Лиувиллю. При $\alpha_{4}=\alpha_{5}=0$ гамильтониан (1.7) хорошо известен [3]. В случае, когда $\alpha_{1}=\alpha_{2}=\alpha_{3}=\alpha_{6}=0$, гамильтониан (1.7) определяет новый частично интегрируемый (т.е. интегрируемый на специальном уровне одного из 
интегралов движения) случай для уравнений Кирхгофа. Переменные и кривую разделения для модели (1.7) можно найти в разделе 4.

Там же приведены (возможно) новые интегрируемые деформации цепочки Тоды и модели Гейзенберга. Нам не известно, существуют ли физические приложения для этих интегрируемых систем. Кроме того, в разделе 4 показано, что цепочки Тоды, соответствуюшие системам корней $B_{N}^{(1)}, C_{N}^{(1)}, A_{2 N}^{(2)}, A_{2 N+1}^{(2)}$ и $D_{N+1}^{(2)}$, а также деформация цепочки Тоды, найденная Иноземцевым в работе [5], допускают разделение в одном и том же наборе переменных разделения, описанном в нашей схеме.

\section{2. СВОЙСТВА СКОБОК СКЛЯНИНА}

2.1. Первые скобки. Явная форма скобок Склянина для переменных (1.3) может быть извлечена из операторного определения

$$
\{\stackrel{1}{T}(\lambda) \stackrel{2}{T}(\mu)\}=[r(\lambda-\mu), \stackrel{1}{T}(\lambda) \stackrel{2}{T}(\mu)] .
$$

Здесь мы используем стандартные обозначения $\stackrel{1}{T}(\lambda)=T(\lambda) \otimes \mathrm{Id}, \stackrel{2}{T}(\mu)=\operatorname{Id} \otimes T(\mu)$ и

$$
r(z)=\frac{\eta}{z} \Pi, \quad \Pi=\left(\begin{array}{llll}
1 & 0 & 0 & 0 \\
0 & 0 & 1 & 0 \\
0 & 1 & 0 & 0 \\
0 & 0 & 0 & 1
\end{array}\right), \quad \eta \in \mathbb{C} .
$$

ПримеР. В простейшем случае $N=2$ покомпонентная запись соотношения (2.1) имеет вид

$$
\begin{aligned}
& \left\{A_{1}, A_{0}\right\}=0, \quad\left\{A_{1}, B_{1}\right\}=\eta B_{1}, \quad\left\{A_{1}, C_{1}\right\}=-\eta C_{1}, \quad\left\{A_{1}, B_{0}\right\}=\eta B_{0}, \\
& \left\{A_{1}, C_{0}\right\}=-\eta C_{0}, \quad\left\{A_{1}, D_{0}\right\}=0, \quad\left\{A_{0}, B_{1}\right\}=\eta B_{0}, \quad\left\{A_{0}, C_{1}\right\}=-\eta C_{0}, \\
& \left\{A_{0}, B_{0}\right\}=\eta\left(B_{0} A_{1}-A_{0} B_{1}\right), \quad\left\{A_{0}, C_{0}\right\}=\eta\left(A_{0} C_{1}-C_{0} A_{1}\right), \\
& \left\{A_{0}, D_{0}\right\}=\eta\left(B_{0} C_{1}-B_{1} C_{0}\right), \quad\left\{B_{1}, C_{1}\right\}=\left\{B_{1}, B_{0}\right\}=0, \\
& \left\{B_{1}, C_{0}\right\}=-\eta D_{0}, \quad\left\{B_{1}, D_{0}\right\}=0, \quad\left\{B_{0}, C_{1}\right\}=-\eta D_{0}, \quad\left\{B_{0}, C_{0}\right\}=-\eta D_{0} A_{1}, \\
& \left\{B_{0}, D_{0}\right\}=-\eta B_{1} D_{0}, \quad\left\{C_{1}, C_{0}\right\}=0, \quad\left\{C_{1}, D_{0}\right\}=0, \quad\left\{C_{0}, D_{0}\right\}=\eta C_{1} D_{0} .
\end{aligned}
$$

ЛЕмма 1 [1]. Для любой числовой матрицы $K$, не зависящей от спектрального параметра, коэффичиенты следа матрицы $K T(\lambda)$ коммутируют друг с другом относительно первых скобок Склянина.

Таким образом, коэффициенты следа матрицы $K T(\lambda)$ порождают коммутативную подалгебру $\mathfrak{A}_{N}^{0}$ в $\mathfrak{A}_{N}$, элементы которой являются линейными многочленами от переменных (1.3).

Коэффициенты определителя

$$
d(\lambda)=\operatorname{det} T(\lambda)=A(\lambda) D(\lambda)-B(\lambda) C(\lambda)
$$


принадлежат центру алгебры $\mathfrak{A}$ или, другими словами, являются функциями Казимира для скобок (2.1). Число функций Казимира равно $2 N-1$, и мы имеем $(4 N-1)$-мерное пуассоново многообразие с вырожденной пуассоновой структурой $(2.1)$ и $2 N$-мерными симплектическими листами общего положения.

Для приведения скобок Пуассона (2.1) к канонической форме можно использовать набор переменных

$$
d_{0}, \ldots, d_{2 N-2}, \quad q_{1}, \ldots q_{N}, \quad p_{1}, \ldots, p_{N},
$$

предложенных Скляниным в работе [2]. Переменные $d_{0}, \ldots, d_{2 N-2}-$ это коэффициенты $(2.4)$

$$
d(\lambda)=d_{2 N-2} \lambda^{2 N-2}+\cdots+d_{0}
$$

переменные $q_{i}$ определяются нулями многочлена

$$
A(\lambda)=\prod_{j=1}^{N}\left(\lambda-q_{j}\right)
$$

переменные $p_{i}$ задаются формулой

$$
p_{j}=\eta^{-1} \ln B\left(q_{j}\right)
$$

Из (2.1) следует, что

$$
\begin{aligned}
& \{A(\lambda), A(\mu)\}=\{B(\lambda), B(\mu)\}=0 \\
& \{A(\lambda), B(\mu)\}=\frac{\eta}{\lambda-\mu}(A(\lambda) B(\mu)-A(\mu) B(\lambda))
\end{aligned}
$$

откуда нетрудно получить $\left\{q_{j}, q_{k}\right\}=\left\{p_{j}, p_{k}\right\}=0$ и $\left\{p_{j}, q_{k}\right\}=\delta_{j k}$. Как обычно, если мы фиксируем значения функций Казимира $d_{j}$, то канонически сопряженные переменные $q_{i}, p_{i}$ становятся симплектическими координатами на соответствующем симплектическом листе.

Исходные переменные (1.3) выражаются через переменные (2.5) с помошью формул

$$
\begin{gathered}
A(\lambda)=\prod_{j=1}^{N}\left(\lambda-q_{j}\right), \quad B(\lambda)=\sum_{j=1}^{N} e^{\eta p_{j}} \prod_{k \neq j}^{N}\left(\frac{\lambda-q_{k}}{q_{j}-q_{k}}\right), \\
C(\lambda)=-\sum_{j=1}^{N} d\left(q_{j}\right) e^{-\eta p_{j}} \prod_{k \neq j}^{N}\left(\frac{\lambda-q_{k}}{q_{j}-q_{k}}\right), \quad D(\lambda)=\frac{d(\lambda)+B(\lambda) C(\lambda)}{A(\lambda)} .
\end{gathered}
$$

2.2. Вторые скобки. Вторые скобки Склянина определяются так называемым уравнением отражения

$$
\{\stackrel{1}{T}(\lambda), \stackrel{2}{T}(\mu)\}=[r(\lambda-\mu) \stackrel{1}{T}(\lambda) \stackrel{2}{T}(\mu)]+\stackrel{1}{T}(\lambda) r(\lambda+\mu) \stackrel{2}{T}(\mu)-\stackrel{2}{T}(\mu) r(\lambda+\mu) \stackrel{1}{T}(\lambda),
$$

где $r(z)$ задается формулой $(2.2)$. 
ЛЕмма 2 [6]. Если матрица $T(\lambda)$ удовлетворяет уравнению отражсения (2.7), то для любой числовой матрищъц $K$ вида

$$
K(\lambda)=\left(\begin{array}{cc}
\alpha_{1} \lambda+\alpha_{0} & \beta_{1} \lambda \\
\gamma_{1} \lambda & -\alpha_{1} \lambda+\alpha_{0}
\end{array}\right)
$$

где $\alpha_{0}, \alpha_{1}, \beta_{1}, \gamma_{1}$ - произвольные постоянные, коэффициенты следа матрицы $K(\lambda) T(\lambda)$ коммутируют между собой относительно второй скобки Склянина.

Канонические переменные

$$
d_{0}, \ldots, d_{2 N}, \quad q_{1}, \ldots q_{N}, \quad p_{1}, \ldots, p_{N}
$$

для скобки Пуассона (2.7) были предложены в работе [2]. Функции Казимира $d_{0}, \ldots, d_{2 N}$ являются коэффициентами определителя (2.4)

$$
d(\lambda)=-\lambda^{4 N+2}+d_{2 N} \lambda^{4 N}+\cdots+d_{1} \lambda^{2}+d_{0},
$$

переменные $q_{i}$ - нули многочлена

$$
C(\lambda)=\lambda \prod_{j=1}^{N}\left(\lambda^{2}-q_{j}^{2}\right)
$$

сопряженные переменные $p_{i}$ задаются формулой

$$
p_{j}=\eta^{-1} \ln A\left(q_{j}\right) \text {. }
$$

2.3. Связь между скобками. Пусть матрица $T$ задана формулами (1.1), (1.2). Рассмотрим матрицу

$$
\mathcal{T}(\lambda)=T(\lambda) K_{-}(\lambda)\left(\begin{array}{cc}
0 & -1 \\
1 & 0
\end{array}\right) T^{t}(-\lambda)\left(\begin{array}{cc}
0 & -1 \\
1 & 0
\end{array}\right)
$$

где $K_{-}(\lambda)$ - произвольная матрица вида (2.8), индекс $t$ означает матричное транспонирование. Нетрудно проверить, что элементы $\mathcal{T}(\lambda)$ имеют структуру (1.4). Формула (2.10) выражает коэффициенты (1.5) матрицы $\mathcal{T}(\lambda)$ через коэффициенты (1.3) матрицы $T$. Оказывается [6], что отображение $\Psi$ согласовано со скобками Склянина: $\Psi\left(\{f, g\}_{2}\right)=\{\Psi(f), \Psi(g)\}_{1}$ для любых функций $f$ и $g$ от переменных (1.5). 


\section{3. КОММУТАТИВНЫЕ ПОДАЛГЕБРЫ}

3.1. Первые скобки. Рассмотрим матрицу

$$
\widetilde{T}(\lambda)=K(\lambda) T(\lambda),
$$

где $T(\lambda)$ задано формулами (1.1) и (1.2), а элементы матрищы

$$
K(\lambda)=\left(\begin{array}{cc}
\mathcal{A} & \mathcal{B} \\
\mathcal{C} & 0
\end{array}\right)(\lambda)
$$

имеют следуюшую структуру:

$$
\begin{gathered}
\mathcal{A}(\lambda)=\mathcal{A}_{M} \lambda^{M}+\mathcal{A}_{M-1} \lambda^{M-1}+\cdots+\mathcal{A}_{0}, \\
\mathcal{B}(\lambda)=b_{M} \lambda^{M}+b_{M-1} \lambda^{M-1}+\cdots+b_{0}, \quad \mathcal{C}(\lambda)=c_{M} \lambda^{M}+c_{M-1} \lambda^{M-1}+\cdots+c_{0},
\end{gathered}
$$

где $b_{k}, c_{k} \in \mathbb{C}$ - произвольные параметры.

Потребуем, чтобы след новой матрицы $\widetilde{T}$ имел вид

$$
\operatorname{tr} \widetilde{T}(\lambda)=\sum_{i=N}^{N+M} a_{i-N} \lambda^{i}+\sum_{k=0}^{N-1} I_{k} \lambda^{k}, \quad a_{i} \in \mathbb{C},
$$

где $a_{i}$ - произвольные постоянные параметры. Легко проверить, что неизвестные коэффициенты $\mathcal{A}_{i}$ многочлена $\mathcal{A}$ и неизвестные функции $I_{k}$ в (3.3) однозначно определяются из (3.3) как функции от динамических переменных (1.3) и параметров $a_{i}, b_{i}$ и $c_{i}$. Эти функции находятся последовательно приравниванием коэффишиентов при степенях $\lambda$ в (3.3) и оказываются многочленами от переменных (1.3) такими, что степени $\mathcal{A}_{i}$ равны $M-i$, а все многочлены $I_{k}$ имеют одну и ту же степень $M+1$.

ПРИмЕР (продолжение). При $N=2$ и $M=1$ многочлены $\mathcal{A}_{i}$ и $I_{i}$ имеют вид

$$
\begin{aligned}
\mathcal{A}_{1} & =a_{1}, \quad \mathcal{A}_{0}=a_{0}-a_{1} A_{1}-b_{1} C_{1}-c_{1} B_{1}, \\
I_{1} & =a_{1}\left(A_{0}-A_{1}^{2}\right)+b_{1}\left(C_{0}-A_{1} C_{1}\right)+c_{1}\left(B_{0}-A_{1} B_{1}\right)+a_{0} A_{1}+b_{0} C_{1}+c_{0} B_{1}, \\
I_{0} & =\left(a_{0}-a_{1} A_{1}-b_{1} C_{1}-c_{1} B_{1}\right) A_{0}+c_{0} B_{0}+b_{0} C_{0} .
\end{aligned}
$$

Теорема 1. Определенные формулой (3.3) многочлены $I_{k}, k=1, \ldots, N$, от переменных (1.3) коммутируют друг с другом относительно скобки (2.1).

ДокАЗАТЕЛЬСтво. Соотношение (3.3) имеет вид

$$
\mathcal{A}(\lambda) A(\lambda)+\mathcal{B}(\lambda) C(\lambda)+\mathcal{C}(\lambda) B(\lambda)=\sum_{k=0}^{N-1} I_{k} \lambda^{k}+\sum_{i=N}^{N+M} a_{i-N} \lambda^{i} .
$$

Подставим $\lambda=q_{j}$ в это тождество. Тогда из соотношения $d(\lambda)=A D-B C$ следует, что

$$
C\left(q_{j}\right)=-\frac{d\left(q_{j}\right)}{B\left(q_{j}\right)}=-d\left(q_{j}\right) e^{-\eta p_{j}} .
$$


Используя эту формулу и определение переменных $p_{i}$, получаем $N$ линейных уравнений

$$
\mathcal{C}\left(q_{j}\right) e^{\eta p_{j}}-\mathcal{B}\left(q_{j}\right) d\left(q_{j}\right) e^{-\eta p_{j}}=\sum_{k=0}^{N-1} I_{k} q_{j}^{k}+\sum_{i=N}^{N+M} a_{i-N} q_{j}^{i}, \quad j=1, \ldots, N,
$$

для $N$ неизвестных функций $I_{k}$. Следуя работе [7], мы легко покажем, что функции $I_{k}$ находятся в инволюции относительно скобки (2.1). Для этого перепишем систему уравнений (3.5) в матричной форме:

$$
\phi(p, q)=\mathbf{S}(q) I(p, q)+U(q)
$$

где

$$
\begin{gathered}
\phi_{j}=\mathcal{C}\left(q_{j}\right) e^{\eta p_{j}}-\mathcal{B}\left(q_{j}\right) d\left(q_{j}\right) e^{-\eta p_{j}}, \quad U_{j}=\sum_{i=N}^{N+M} a_{i-N} q_{j}^{i}, \\
\mathbf{S}=\left(\begin{array}{cccc}
1, & q_{1}, & \ldots & q_{1}^{N-1} \\
\vdots & \vdots & \ddots & \vdots \\
1, & q_{N}, & \ldots & q_{N}^{N-1}
\end{array}\right)
\end{gathered}
$$

Решение системы (3.6) задается формулой

$$
I(p, q, d)=\mathbf{W}(q)(\phi(p, q)-U(q)), \quad \mathbf{W}(q)=\mathbf{S}^{-1}(q) .
$$

Матрища $\mathbf{S}$ принадлежит к классу так называемых штекелевых матриц [7], для которых элементы $S_{i j}$ строки с номером $i$ зависят только от переменной $q_{i}$. Дифференцируя тождество

$$
\sum_{i} W_{m i} S_{i l}=\delta_{m l}
$$

по переменной $q_{j}$ и используя основное свойство штекелевых матриц, получим

$$
\sum_{i} \frac{\partial W_{m i}}{\partial q_{j}} S_{i l}=-W_{m j} \frac{\partial S_{j l}}{\partial q_{j}}
$$

Подставляя $k$ вместо $m$, получим еше одно соотношение того же вида. Исключая выражение $\partial S_{j l} / \partial q_{j}$ из этих двух уравнений, находим

$$
\sum_{i}\left(W_{j k} \frac{\partial W_{i m}}{\partial q_{j}}-W_{j m} \frac{\partial W_{i k}}{\partial q_{j}}\right) S_{i l}=0 .
$$

Так как определитель матрицы $\mathbf{S}$ не равен нулю, имеем

$$
W_{j k} \frac{\partial W_{i m}}{\partial q_{j}}-W_{j m} \frac{\partial W_{i k}}{\partial q_{j}}=0
$$

для всех $j, k, i, m$.

Вычисляя теперь скобки Пуассона между функциями $I_{k}$ и $I_{m}(3.7)$, получаем

$$
\left\{I_{k}, I_{m}\right\}=\sum_{i, j}\left(W_{j k} \frac{\partial W_{i m}}{\partial q_{j}}-W_{j m} \frac{\partial W_{i k}}{\partial q_{j}}\right) \frac{\partial \phi_{j}}{\partial p_{j}}\left(\phi_{j}-U_{j}\left(q_{j}\right)\right) .
$$

Из (3.8) следует, что $\left\{I_{k}, I_{m}\right\}=0$. Доказательство завершено. 
СлЕДСТВИЕ 1. Функиии $q_{j}, p_{j}$ являются переменными разделения для интегралов движения $I_{k}$, удовлетворяющих разделяющим уравнениям (3.5), (3.6). Переменные

$$
\lambda_{j}=q_{j}, \quad \mu_{j}=\mathcal{C}\left(q_{j}\right) e^{\eta p_{j}}
$$

удовлетворяют характеристическому уравнению $\operatorname{Det}(\widetilde{T}(\lambda)-\mu)=0$. Это значит, что все пары переменных $\lambda_{j}, \mu_{j}$ лежсат на одной и той жсе алгебраической кривой.

ЗАмечАние 1 . В случае $N=2, M=1$ матрица $\widetilde{T}(\lambda)$ вида $(3.1)$ с

$$
K(\lambda)=\left(\begin{array}{cc}
\lambda+\mathcal{A}_{0} & b_{0} \\
c_{0} & 0
\end{array}\right), \quad \mathcal{A}_{0}=a_{0}-A_{1},
$$

появилась в статье [1] в нефакторизованном виде. В работе [8] эта матрица была факторизована и обобшена на случай произвольного $N$. Отметим, что наша матрица $K$ для $M=1$

$$
K(\lambda)=\left(\begin{array}{cc}
\lambda+\mathcal{A}_{0} & b_{1} \lambda+b_{0} \\
c_{1} \lambda+c_{0} & 0
\end{array}\right), \quad \mathcal{A}_{0}=a_{0}-A_{1}-b_{1} C_{1}-c_{1} B_{1},
$$

имеет более обшую форму, чем (3.10). Параметры $b_{1}$ и $c_{1}$ чрезвычайно сушественны для построения новых квадратичных интегрируемых гамильтонианов.

ЗАМЕчАНИЕ 2. Обобщая условие (3.3), можно предположить, что

$$
\operatorname{tr} \widetilde{T}(\lambda)=\sum_{i \in \widehat{\varrho}} a_{i} \lambda^{i}+\sum_{k \in \varrho} I_{k} \lambda^{k}, \quad a_{k} \in \mathbb{C} .
$$

Здесь $\varrho=\left\{i_{1}, \ldots, i_{N}\right\}$ - произвольное подмножество множества чисел $\{0,1, \ldots, N+$ $M\}$, а $\widehat{\varrho}$ - соответствуюшее дополнение: $\varrho \cup \widehat{\varrho}=\{0,1, \ldots, N+M\}$. В этом случае $I_{i}$ по-прежнему коммутируют между собой, но являются рациональными функциями переменных (1.3)

ПримеР (продолжение). Если $N=2, M=1$ и $\operatorname{tr} \widetilde{T}(\lambda)=a_{1} \lambda^{3}+I_{1} \lambda^{2}+a_{0} \lambda+I_{0}$, то

$$
\begin{aligned}
& \mathcal{A}_{1}=a_{1}, \quad \mathcal{A}_{0}=\frac{1}{A_{1}}\left(a_{0}-a_{1} A_{0}-c_{0} B_{1}-b_{0} C_{1}-b_{1} C_{0}-c_{1} B_{0}\right), \\
& I_{1}=\frac{1}{A_{1}}\left(a_{0}-a_{1}\left(A_{0}-A_{1}^{2}\right)-b_{1}\left(C_{0}-C_{1} A_{1}\right)-c_{1}\left(B_{0}-B_{1} A_{1}\right)-c_{0} B_{1}-b_{0} C_{1}\right) \\
& I_{0}=\frac{1}{A_{1}}\left(A_{0}\left(a_{0}-c_{1} B_{0}-a_{1} A_{0}-b_{1} C_{0}\right)+b_{0}\left(C_{0} A_{1}-A_{0} C_{1}\right)+c_{0}\left(B_{0} A_{1}-A_{0} B_{1}\right)\right) .
\end{aligned}
$$

ЗАмЕчаниЕ 3. Доказательство теоремы 1 может быть легко модифицировано на случай матриц (1.1), для которых матричный коэффициент при старшей степени $\lambda$ имеет не жорданову, а диагональную структуру. А именно можно предположить, что элемент $D(\lambda)$ матрицы $T(\lambda)$ имеет вид

$$
D(\lambda)=\lambda^{N}+D_{N-1} \lambda^{N-1}+D_{N-2} \lambda^{N-2}+\cdots+D_{0}
$$

В этом случае имеются одна дополнительная переменная $D_{N-1}$ и одна дополнительная функция Казимира $d_{2 N-1}=A_{N-1}+D_{N-1}$. 
3.2. Вторые скобки. Конструкция коммутативных подалгебр для вторых скобок очень близка к изложенной выше. Пусть матрица $T(\lambda)$ задана формулами $(1.1),(1.4)$ и удовлетворяет уравнению отражения (2.7). Введем матрицу $\widetilde{T}=K T(3.1)$, где элементы матрицы

$$
K(\lambda)=\left(\begin{array}{cc}
\mathcal{A} & \mathcal{B} \\
0 & \mathcal{D}
\end{array}\right)(\lambda)
$$

имеют вид

$$
\begin{gathered}
\mathcal{A}(\lambda)=a_{2 M} \lambda^{2 M}+a_{2 M-1} \lambda^{2 M-1}+\cdots+a_{1} \lambda+a_{0}, \quad \mathcal{D}(\lambda)=\mathcal{A}(-\lambda) \\
\mathcal{B}(\lambda)=\mathcal{B}_{M-1} \lambda^{2 M-1}+\mathcal{B}_{M-2} \lambda^{2 M-3}+\cdots+\mathcal{B}_{0} \lambda
\end{gathered}
$$

Здесь $a_{k} \in \mathbb{C}$ - произвольные параметры, а коэффициенты $\mathcal{B}_{i}-$ некоторые функции от переменных (1.5). Нетрудно проверить, что след матришы $\widetilde{T}(\lambda)$ является четным многочленом от $\lambda$.

Функции $\mathcal{B}_{0}, \ldots, \mathcal{B}_{M-1}$ и $I_{0}, \ldots I_{N-1}$ однозначно определяются требованием

$$
\operatorname{tr} \widetilde{T}(\lambda)=\sum_{i=N+1}^{N+M} b_{i-N} \lambda^{2 i}+\sum_{k=1}^{N} I_{k} \lambda^{2 k}+a_{0} d_{0}, \quad b_{i} \in \mathbb{C}
$$

где $b_{i}$ - произвольные фиксированные числовые параметры, $d_{0}=2 A_{0}$ - функция Казимира (2.9). Все функции $\mathcal{B}_{i}, I_{i}$ оказываются многочленами по переменным (1.5), например, $\mathcal{B}_{M-1}=b_{M}-2 a_{2 M} A_{2 N}$.

Те же самые рассуждения, что и при доказательстве теоремы 1 , приводят к следующему утверждению.

ТеОрема 2. Определенные соотношением (3.14) многочлены $I_{k}$ от переменных (1.5) коммутируют между собой относительно скобки (2.7).

СлеДСТВИЕ 2. Канонические переменные $p_{i}, q_{i}(2.9)$ являются переменными разделения для интегралов движения $I_{k}(3.14)$. Разделяющие уравнения задаются формулой (3.6), где

$$
\begin{gathered}
\phi_{j}=\mathcal{A}\left(q_{j}\right) e^{\eta p_{j}}-\mathcal{D}\left(q_{j}\right) d\left(q_{j}\right) e^{-\eta p_{j}}, \\
\mathbf{S}=\left(\begin{array}{cccc}
q_{1}^{2}, & q_{1}^{4}, & \cdots & U_{j}=\sum_{i=1}^{M N} b_{i} q_{j}^{2(N+i)}+a_{0} d_{0} \\
\vdots & \vdots & \ddots & \vdots \\
q_{N}^{2}, & q_{N}^{4}, & \cdots & q_{N}^{2 N}
\end{array}\right)
\end{gathered}
$$

Переменные

$$
\lambda_{j}=q_{j}, \quad \mu_{j}=\mathcal{A}\left(q_{j}\right) e^{\eta p_{j}}
$$

удовлетворяют характеристическому уравнению $\operatorname{Det}(\widetilde{T}(\lambda)-\mu)=0$. 
ЗАмЕчАнИЕ 4 . В случае, когда параметр $\gamma_{1}$ в формуле (2.8) равен нулю, утверждение леммы 2 совпадает с частным случаем $M=1, a_{2}=0$ теоремы 2.

ЗАмечАниЕ 5. Теорема 2 остается верной, если предположить, что

$$
\operatorname{tr} \widetilde{T}(\lambda)=\sum_{i \in \widehat{\varrho}} b_{i} \lambda^{2 i}+\sum_{k \in \varrho} I_{k} \lambda^{2 k}+a_{0} d_{0}, \quad b_{i} \in \mathbb{C}
$$

где $\varrho=\left\{i_{1}, \ldots, i_{N}\right\}$ - произвольное подмножество множества чисел $\{1, \ldots, N+M\}$, а $\varrho$ - дополнение к $\varrho$. Однако в этом случае $\mathcal{B}_{i}$ и $I_{i}$ становятся рациональными функциями переменных (1.5).

ЗАмЕчаниЕ 6 . Теорема 2 справедлива также для матриц (1.1), у которых элемент $B(\lambda)$ имеет вид

$$
\begin{gathered}
B(\lambda)=\lambda^{2 N+1}+B_{N-1} \lambda^{2 N-1}+B_{N-2} \lambda^{2 N-3}+\cdots+B_{0} \lambda . \\
\text { 4. ПОЛИНОМИАЛЬНЫЕ ДЕФОРМАЦИИ } \\
\text { ИЗВЕСТНЫХ ИНТЕГРИРУЕМЫХ МОДЕЛЕЙ }
\end{gathered}
$$

\section{4. ПОЛИНОМИАЛЬНЫЕ ДЕФОРМАЦИИ}

Если для заданного пуассонова многообразия $\mathcal{M}$ отождествить скобки Пуассона на $\mathcal{M}$ со скобками Склянина (2.1) или (2.7), то определения (2.1) и (2.7) можно рассматривать как уравнения для матриц $T(\lambda)$. Всякое решение этого уравнения представляет собой набор формул, выражающих переменные (1.3) или (1.5) как функции от координат на $\mathcal{M}$. Эти формулы определяют представление алгебры Склянина (2.1) и (2.7) на многообразии $\mathcal{M}$.

4.1. Обобщенный волчок Горячева-Чаплыгина. Орбиты коприсоединенного представления группы движений трехмерного евклидова пространства $E(3)$ на $e(3)^{*}$ со скобкой Ли-Пуассона (1.6) являются четырехмерными симплектическими листами, представляющими собой общий уровень двух функций Казимира $(x, x)=\sum x_{i}^{2}$ и $\ell=$ $\sum x_{i} J_{i}$

Фазовое пространство для волчка Горячева-Чаплыгина $\mathcal{M}$ отождествляется с семейством симплектических листов, выделенных нулевым значением второй функции Казимира $\ell=0$ (интеграла плошадей).

Следуя работе [3], рассмотрим переменные (1.3)

$$
\begin{gathered}
A_{1}=-2 J_{3}, \quad A_{0}=-J_{1}^{2}-J_{2}^{2}-\frac{\delta}{x_{3}^{2}}, \quad B_{1}=x_{2}+i x_{1}, \quad B_{0}=-\left(J_{2}+i J_{1}\right) x_{3}, \\
C_{1}=-x_{2}+i x_{1}, \quad C_{0}=\left(J_{2}-i J_{1}\right) x_{3}, \quad D_{0}=x_{3}^{2}
\end{gathered}
$$

которые удовлетворяют соотношениям (2.3) с $\eta=-2 i$ при условии, что $\ell=0$, а левые части (2.3) вычисляются с помошью скобок Пуассона (1.6). 
Формулы (3.4) дают

$$
\begin{aligned}
I_{1}= & a_{1}\left(J_{1}^{2}+J_{2}^{2}+4 J_{3}^{2}+\frac{\delta}{x_{3}^{2}}\right)+2 a_{0} J_{3}+b_{0}\left(x_{2}-i x_{1}\right)-c_{0}\left(x_{2}+i x_{1}\right)+ \\
& +b_{1}\left(2 J_{3} x_{2}-J_{2} x_{3}-2 i J_{3} x_{1}+i J_{1} x_{3}\right)-c_{1}\left(2 J_{3} x_{2}-J_{2} x_{3}+2 i J_{3} x_{1}-i J_{1} x_{3}\right), \\
I_{0}= & 2 a_{1}\left(J_{1}^{2}+J_{2}^{2}\right) J_{3}+a_{0}\left(J_{1}^{2}+J_{2}^{2}\right)+b_{0}\left(-J_{2}+i J_{1}\right) x_{3}+c_{0}\left(J_{2}+i J_{1}\right) x_{3}+ \\
& +b_{1}\left(J_{1}^{2}+J_{2}^{2}\right)\left(x_{2}-i x_{1}\right)-c_{1}\left(J_{1}^{2}+J_{2}^{2}\right)\left(x_{2}+i x_{1}\right)+ \\
& +\frac{\delta}{x_{3}^{2}}\left(2 a_{1} J_{3}+a_{0}+b_{1}\left(x_{2}-i x_{1}\right)-c_{1}\left(x_{2}+i x_{1}\right)\right) .
\end{aligned}
$$

Согласно теореме 1 интегралы $I_{1}$ и $I_{0}$ находятся в инволюции. При $a_{1}=1$ функция Гамильтона $H_{1}=I_{1}$ после канонического преобразования переменных

$$
x \rightarrow x, \quad J \rightarrow J+U x, \quad U=\left(\begin{array}{ccc}
0 & 0 & -i c_{+} \\
0 & 0 & -c_{-} \\
i c_{+} & c_{-} & 0
\end{array}\right), \quad c_{ \pm}=\frac{c_{1} \pm b_{1}}{2},
$$

и замены параметров

$$
\begin{gathered}
\alpha_{1}=i\left(a_{0}\left(c_{1}+b_{1}\right)-\left(c_{0}+b_{0}\right)\right), \quad \alpha_{2}=\left(a_{0}\left(c_{1}-b_{1}\right)-\left(c_{0}-b_{0}\right)\right), \\
\alpha_{3}=2 a_{0}, \quad 2 \alpha_{5}=c_{1}-b_{1}, \quad 2 \alpha_{4}=i\left(c_{1}+b_{1}\right), \quad \alpha_{6}=\delta
\end{gathered}
$$

совпадает с гамильтонианом (1.7).

Приведем более обшую деформацию гамильтониана Горячева-Чаплыгина, соответствуюшую $M=2$. Она кубична по моментам $J_{i}$ и имеет вид

$$
\begin{aligned}
\hat{I}_{1}= & I_{1}+4 a_{2} J_{3}\left(\left(J_{1}^{2}+J_{2}^{2}+2 J_{3}^{2}\right)+\delta x_{3}^{-2}\right)+ \\
& +b_{2}\left(2 i J_{3} x_{3}\left(J_{1}+i J_{2}\right)+\left(J_{1}^{2}+J_{2}^{2}+4 J_{3}^{2}+\delta x_{3}^{-2}\right)\left(x_{2}-i x_{1}\right)\right)- \\
& -c_{2}\left(2 i J_{3} x_{3}\left(J_{1}-i J_{2}\right)+\left(J_{1}^{2}+J_{2}^{2}+4 J_{3}^{2}+\delta x_{3}^{-2}\right)\left(x_{2}+i x_{1}\right)\right)
\end{aligned}
$$

где $I_{1}$ задается формулой (4.1).

Простейший рациональный интегрируемый гамильтониан, который можно построить с помощью схемы из п. 3.1, задается формулой (3.11) и имеет вид

$$
\tilde{I}_{1}=\frac{I_{1}-a_{0}\left(2 J_{3}-1\right)}{2 J_{3}} .
$$

Дополнительный интеграл $\tilde{I}_{0}$ определяется последней из формул (3.11).

Согласно теореме 1 и следствию 1 , переменные разделения для всех деформаций одни и те же. В соответствии с определением (2.5) координаты $q_{1,2}$ являются нулями многочлена $A(\lambda)=\lambda^{2}+A_{1} \lambda+A_{0}$, т.е.

$$
q_{1,2}=J_{3} \pm \sqrt{J_{1}^{2}+J_{2}^{2}+J_{3}^{2}+\frac{\delta}{x_{3}^{2}}}
$$


Соответствующие импульсы $p_{1,2}$ определяются формулой (2.6):

$$
p_{1,2}=\frac{1}{2 i} \ln \left(q_{1,2}\left(i x_{1}-x_{2}\right)-\left(i J_{1}-J_{2}\right) x_{3}\right) .
$$

Уравнения разделения (3.5) и соответствуюшие детерминантные кривые, связываюшие переменные (3.9), для разных деформаций различны. Например, для интегралов (4.1), (4.2) детерминантная кривая имеет вид

$$
a_{1} \lambda^{3}+a_{0} \lambda^{2}-I_{1} \lambda+I_{0}=\left(c_{1} \lambda+c_{0}\right) \mu-\frac{\left(b_{1} \lambda+b_{0}\right) \lambda^{2}(x, x)}{\mu} .
$$

Для рассмотренного выше рационального случая кривая отличается перестановкой местами параметра $a_{0}$ и интеграла движения $I_{1}$ :

$$
a_{1} \lambda^{3}+\tilde{I}_{1} \lambda^{2}-a_{0} \lambda+\tilde{I}_{0}=\left(c_{1} \lambda+c_{0}\right) \mu-\frac{\left(b_{1} \lambda+b_{0}\right) \lambda^{2}(x, x)}{\mu} .
$$

\section{2. Цепочки Тоды.}

4.2.1. Первые скобки. Введем канонические координаты $p_{i}, q_{i},\left\{p_{i}, q_{k}\right\}=\delta_{i k}$ на фазовом пространстве $\mathcal{M}=\mathbb{R}^{2 N}$ и определим представление на $\mathcal{M}$ алгебры Склянина (2.1) с $\eta=1$ с помошью формулы (см. [9])

$$
T(\lambda)=L_{N} L_{N-1} \ldots L_{1}, \quad L_{i}(\lambda)=\left(\begin{array}{cc}
\lambda-p_{i} & e^{q_{i}} \\
-e^{-q_{i}} & 0
\end{array}\right) .
$$

Известно, что коэффициенты следа матрицы $T(\lambda)$ порождают интегралы движения для периодической цепочки Тоды:

$$
P=-\sum_{i=1}^{N} p_{i}, \quad H=-\sum_{i>j}^{N} p_{i} p_{j}-\sum_{i=1}^{N} e^{q_{i+1}-q_{i}}, \quad q_{i+N}=q_{i} .
$$

Взаимодействие становится локальным, если вместо $H$ рассмотреть гамильтониан $H+$ $P^{2} / 2$.

Используя схему, предложенную в разделе 3 , можно построить новые интегрируемые системы с полиномиальными по импульсам интегралами движения. В простейшем случае $M=1$ коммутативная подалгебра, построенная в п. 3.1, содержит квадратичный интеграл

$$
I_{N-1}=a_{0} P+a_{1}\left(H-P^{2}\right)+e^{q_{1}}\left(c_{0}+c_{1} p_{1}\right)-e^{-q_{N}}\left(b_{0}+b_{1} p_{N}\right) .
$$

Эта модель может рассматриваться как деформация открытой цепочки Тоды, соответствуюшая случаю $a_{0}=c_{0}=c_{1}=b_{0}=b_{1}=0$. Заметим, что если одна из постоянных $b_{i}$ или $c_{i}$ отлична от нуля, то модель не допускает линейного интеграла $P$ и, в отличие от цепочки Тоды, мы не можем с помощью сдвига на $P^{2}$ сделать локальной кинетическую энергию. 
4.2.2. Вторые скобки. Пусть $\mathcal{T}(\lambda)$ - частное решение уравнения отражения вида (2.10):

$$
\mathcal{T}(\lambda)=T(\lambda)\left(\begin{array}{cc}
\alpha_{1} \lambda+\alpha_{0} & 0 \\
\lambda & -\alpha_{1} \lambda+\alpha_{0}
\end{array}\right)\left(\begin{array}{cc}
0 & -1 \\
1 & 0
\end{array}\right) T^{t}(-\lambda)\left(\begin{array}{cc}
0 & -1 \\
1 & 0
\end{array}\right)
$$

где $T$ задана формулой (4.3).

Рассмотрим теперь матрицу $\widetilde{\mathcal{T}}(\lambda)=K(\lambda) \mathcal{T}(\lambda)(3.1)$ при $M=1$; тогда (см. $(3.13))$

$$
K(\lambda)=\left(\begin{array}{cc}
a_{2} \lambda^{2}+a_{1} \lambda+a_{0} & \mathcal{B}_{1} \lambda \\
0 & a_{2} \lambda^{2}-a_{1} \lambda+a_{0}
\end{array}\right) .
$$

Из условия, что след матрищы $\widetilde{\mathcal{T}}(\lambda)$ порождает интегралы движения (3.14), находим, что динамическая матрица $K$ зависит только от координаты в $N$-м узле решетки

$$
\mathcal{B}_{1}=b_{1}-2 a_{2} e^{-q_{N}}
$$

где без ограничения общности можно положить $b_{1}=1$. При условии $a_{2}=0$ эта матрица не зависит от динамических переменных, и справедлива лемма 2.

Так, если все параметры $a_{i}=0$, то $\mathcal{B}_{1}=1$ и с точностью до канонического преобразования

$$
p_{1} \rightarrow p_{1}-\alpha_{1} e^{q_{1}}
$$

гамильтониан равен

$$
H=\sum_{i=1}^{N} \frac{p_{i}^{2}}{2}+\sum_{i=1}^{N-1} e^{q_{i+1}-q_{i}}-\alpha_{0} e^{q_{1}}-\frac{\alpha_{1}^{2}}{2} e^{2 q_{1}}
$$

Гамильтониан $H$ описывает цепочки Тоды, связанные с классическими системами корней типа $B_{N}\left(\right.$ при $\left.\alpha_{1}=0\right)$ и $C_{N}$ (при $\left.\alpha_{0}=0\right)$ [6], [10].

В общем случае $a_{i} \neq 1$ матрица $\tilde{\mathcal{T}}(\lambda)=K(\lambda) \mathcal{T}(\lambda)$ порождает гамильтониан вида $H_{a}=H+\Delta H$, где дополнение к функции Гамильтона $H(4.6)$ равно

$$
\Delta H=-\left(a_{2} p_{N}^{2}-a_{1} p_{N}+a_{0}\right) e^{-q_{N}}-a_{2} e^{-q_{N-1}} .
$$

При $a_{2}=0$ после канонического преобразования импульсов

$$
p_{N}=\hat{p}_{N}-a_{1} e^{-q_{N}}
$$

данный гамильтониан имеет вид

$$
H_{a}=\sum_{i=1}^{N} \frac{p_{i}^{2}}{2}+\sum_{i=1}^{N-1} e^{q_{i+1}-q_{i}}-\alpha_{0} e^{q_{1}}-\frac{\alpha_{1}}{2} e^{2 q_{1}}-a_{0} e^{-q_{N}}-\frac{a_{1}^{2}}{2} e^{-2 q_{N}}
$$

и описывает цепочки Тоды, связанные с пополненными системами корней $C_{N}^{(1)}$ при $\alpha_{0}=$ $a_{0}=0, A_{2 N}^{(2)}$ при $\alpha_{1}=a_{0}=0$ и $D_{N+1}^{(2)}$ при $\alpha_{1}=a_{1}=0$. 
При $a_{2} \neq 0$ каноническое преобразование пары координат

$$
p_{N}=\frac{\left(\operatorname{ch} \hat{q}_{N}+1\right) \hat{p}_{N}}{\operatorname{sh} \hat{q}_{N}}-\frac{a_{1}}{a_{2}\left(\operatorname{ch} \hat{q}_{N}-1\right)}, \quad q_{N}=\ln \left(a_{2}\left(\operatorname{ch} \hat{q}_{N}+1\right)\right)
$$

приводит гамильтониан $H_{a}=H+\Delta H$ к натуральному виду

$$
\begin{aligned}
H_{a}= & \sum_{i=1}^{N} \frac{p_{i}^{2}}{2}+\sum_{i=1}^{N-1} e^{q_{i+1}-q_{i}}-\alpha_{0} e^{q_{1}}-\frac{\alpha_{1}}{2} e^{2 q_{1}}+ \\
& +a_{2}\left(e^{\hat{q}_{N}-q_{N-1}}+e^{-\hat{q}_{N}-q_{N}-1}\right)-\frac{a_{1}^{2}}{a_{2}^{2} \operatorname{sh}\left(\hat{q}_{N}\right)^{2}}-\frac{a_{0}}{a_{2} \operatorname{sh}\left(\hat{q}_{N} / 2\right)^{2}}
\end{aligned}
$$

Полученный гамильтониан при $a_{0}=a_{1}=0$ отвечает цепочке Тоды, связанной с корневыми системами $B_{N}^{(1)}$ при $\alpha_{1}=\beta_{1}=0$ и $A_{2 N+1}^{(2)}$ при $\alpha_{1}=\alpha_{0}=0$. Второе и третье добавочные слагаемые были найдены Иноземцевым в работе [5]. Нам не известна интерпретация этих добавок в терминах систем корней.

Все интегрируемые гамильтонианы, возникшие в п. 4.2.2, были известны. В данной работе они построены в рамках единого подхода, который позволил доказать, что цепочки Тоды, отвечающие корневым системам $B_{N}^{(1)}, C_{N}^{(1)}, A_{2 N}^{(2)}, A_{2 N+1}^{(2)}$ и $D_{N+1}^{(2)}$, допускают разделение в одном и том же (с точностью до канонических преобразований (4.5) и (4.7)) наборе переменных разделения.

\section{3. Спиновые модели.}

4.3.1. Первые скобки. Рассмотрим в качестве пространства $\mathcal{M}$ прямую сумму алгебр $s l^{*}(2)$ со скобками

$$
\left\{s_{3}^{i}, s_{ \pm}^{i}\right\}= \pm s_{ \pm}^{i}, \quad\left\{s_{+}^{i}, s_{-}^{i}\right\}=2 s_{3}^{i}
$$

Тогда формула

$$
T(\lambda)=L_{N} L_{N-1} \ldots L_{1}, \quad L_{i}(\lambda)=\left(\begin{array}{cc}
\lambda+s_{3}^{i} & s_{-}^{i} \\
s_{+}^{i} & \lambda-s_{3}^{i}
\end{array}\right)
$$

задает на $\mathcal{M}$ представление алгебры Склянина (2.1) с $\eta=1$ [2]. Нетрудно проверить, что элементы $A, B$ и $C$ такой матрицы $T$ имеют вид (1.2), а элемент $D(\lambda)$ и функция Казимира $d_{2 n-1}=A_{N-1}+D_{N+1}$ равны нулю.

Интегралы, которые получаются с использованием леммы 2, не являются локальными. Однако, раскладывая функцию $\ln (\operatorname{tr} T)$ по степеням $\lambda$, можно получить гамильтониан

$$
H=\sum_{i=1}^{N}\left(2 s_{3}^{i} s_{3}^{i+1}+s_{+}^{i} s_{-}^{i+1}+s_{-}^{i} s_{+}^{i+1}\right)
$$

$X X X$-изотропного магнетика Гейзенберга и остальные интегралы движения для этой модели. Данная функция Гамильтона локальна, т.е. описывает взаимодействие только ближайших узлов в цепочке. 
Дальнейшее построение новых интегрируемых систем с использованием представления скобок Склянина (4.9) проводится по схеме, предложенной в разделе 3 . Однако, так как функции $\mathcal{A}_{i}$ зависят от всех спиновых переменных $s^{i}$ в цепочке, стандартные методы построения интегралов движения приводят к заведомо нелокальным интегралам. Так, при $M=1$ формулы (3.4) дают

$$
\begin{aligned}
\mathcal{A}_{0}= & a_{0}-a_{1} S_{3}-b_{1} S_{+}-c_{1} S_{-} \\
I_{N-1}= & a_{0} S_{3}+b_{0} S_{+}+c_{0} S_{-}+a_{1}\left(\sum_{j>i}^{N}\left(s_{+}^{i} s_{-}^{j}+s_{3}^{i} s_{3}^{j}\right)-S_{3}^{2}\right)- \\
& -2 b_{1}\left(S_{3} S_{+}-\sum_{j>i}^{N} s_{3}^{i} s_{+}^{j}+\frac{1}{2} \sum_{i=1}^{N} s_{3}^{i} s_{+}^{i}\right)- \\
& -2 c_{1}\left(S_{3} S_{-}-\sum_{j<i}^{N} s_{3}^{i} s_{-}^{j}+\frac{1}{2} \sum_{i=1}^{N} s_{3}^{i} s_{-}^{i}\right)
\end{aligned}
$$

где $S_{3}=\sum s_{3}^{i}, S_{-}=\sum s_{-}^{i}, S_{+}=\sum s_{+}^{i}$. Вопрос, можно ли преобразовать этот квадратичньй гамильтониан к более компактному виду, в настояшей работе не обсуждается.

4.3.2. Вторые скобки. Пусть $\mathcal{T}(\lambda)$ - матрица (2.10), где $T(\lambda)$ задана формулой (4.9), $K_{-}$- числовая матрица (2.8). Таким образом, исходное представление алгебры Склянина параметризуется элементами $\alpha_{0}, \alpha_{1}, \beta_{1}$ и $\gamma_{1}$ матрицы (2.8).

Следуя предложенной в разделе 3 схеме, рассмотрим матрицу $\widetilde{\mathcal{T}}(\lambda)=K(\lambda) \mathcal{T}(\lambda)$, где матрица $K(\lambda)$ определена формулой (4.4). Из условия (3.14) находим, что

$$
\mathcal{B}_{1}=-2 a_{2}\left(2 \frac{\alpha_{1}}{\gamma_{1}} S_{3}+\frac{\beta_{1}}{\gamma_{1}} S_{-}+S_{+}+\gamma_{1} \alpha_{0}\right)-2 \frac{\alpha_{1}}{\gamma_{1}} a_{1}+\frac{b_{1}}{\gamma_{1}}
$$

где $S_{ \pm}$и $S_{3}-$ компоненты полного спина (4.10). При условии $a_{2}=0$ эта матрица не зависит от динамических переменных, и мы приходим к частному случаю $\hat{\gamma}_{1}=0$ конструкции, описанной леммой 2.

Коэффициенты следа матрицы $\widetilde{\mathcal{T}}$ приводят к нелокальным интегралам движения. Поскольку матрицы (2.8) и (4.4) являются регулярными, т.е. $K(0) \sim \mathrm{Id}$, для построения локальных функций Гамильтона мы можем использовать формулу Склянина из работы [6]. Так, если $a_{0}=a_{1}=a_{2}=0$, эта формула приводит к гамильтониану

$$
H=\sum_{i=1}^{N-1}\left(2 s_{3}^{i} s_{3}^{i+1}+s_{-}^{i} s_{+}^{i+1}+s_{+}^{i} s_{-}^{i+1}\right)+\frac{1}{\alpha_{0}}\left(\alpha_{1} s_{3}^{1}-\gamma_{1} s_{-}^{1}-\beta_{1} s_{+}^{1}\right)
$$

При $a_{2}=0, a_{1} \neq 0$ возникает дополнение к гамильтониану (4.11) вида

$$
\Delta H=\frac{a_{1}}{a_{0}} s_{3}^{N}-\frac{2 \alpha_{1} a_{1}-b_{1}}{a_{0} \gamma_{1}} s_{+}^{N} .
$$


При $a_{2} \neq 0$ дополнение имеет вид

$$
\Delta H=\frac{2 a_{1} s_{3}^{N}-s_{+}^{N} \mathcal{B}_{1}}{2\left(a_{2}+a_{0}\right)} .
$$

Данное слагаемое нелокально и описывает взаимодействие $N$-го узла цепочки с остальными узлами.

Известно [6], что использование постоянной матрицы $K$ вида $(2.8)$ с $\hat{\gamma}_{1} \neq 0$ позволяет получить еше одно добавочное слагаемое

$$
\Delta H=-\hat{\gamma}_{1} s_{-}^{N},
$$

которое не воспроизводится в рамках предложенной схемы. Заметим, что для цепочек Тоды подобное слагаемое появлялось в нашем методе благодаря наличию канонического преобразования (4.5).

Благодарности. Авторы благодарны Институту математических наук Исаака Ньютона (Кембридж) за гостеприимство. Работа частично поддержана грантами РФФИ № 99-01-00294 и o 99-01-00698; INTAS № 99-1782 и 99-01459; EPSRC GR K99015.

\section{Список литературы}

[1] Е. К. Склянин. Зап. научн. сем. ЛОМИ. 1984. V. 133. Р. 236.

[2] E. K. Sklyanin. Progr. Theor. Phys. 1995. V. 118. P. 35.

[3] V. B. Kuznetsov, A. V. Tsiganov. J. Phys. 1989. V. 22. P. L73.

[4] В. В. Соколов, А. В. Цыганов. ТМФ. 2002. Т. 131. № 1. С. 118.

[5] V. I. Inozemtsev. Commun. Math. Phys. 1989. V. 121. P. 629.

[6] E. K. Sklyanin. J. Phys. A. 1988. V. 21. P. 2375.

[7] P. Stäckel. Über die integration der Hamilton-Jacobischen differentialgeichung mittels separation der variabeln. Halle: Habilitationschrift, 1891; L. A. Pars. Amer. Math. Monthly. 1949. V. 56. P. 394.

[8] A. V. Tsiganov. J. Math. Phys. 1997. V. 38. P. 196

[9] E. K. Sklyanin. The quantum Toda chain. In: Nonlinear Equations in Classical and Quantum Field Theory. Proc. Semin. Meudon, Paris, 1983/1984. Lect. Notes Phys. V. 226. Berlin: Springer, 1985. P. 196.

[10] A. V. Tsiganov. J. Phys. A. 1998. V. 31. P. 8049. 